\title{
Corrosion inhibition of iron surfaces with phosphatidic acid
}

\author{
Ahmed Sameer Al-Badran ${ }^{1,2}$ and Adam Mechler ${ }^{1 \star}$
}

\begin{abstract}
Preventing the corrosion of iron in inaccessible structures requires a coating method that reaches all surface areas and creates a uniform protective layer. An ages old practice to protect iron artefacts is to coat them with animal fat, that is, a mixture of lipids. This "method" is accidentally ingenious: some natural phospholipids found in animal fat have the potential to form a tightly packed self-assembled monolayer on metal oxide surfaces, similar to the surfactant monolayers that have attracted increasing attention lately. Thus, the most primitive corrosion prevention method may point at a way to coat complex iron structures in an industrial environment. Here the ability of phosphatidic acid, a natural lipid, to coat and protect iron surfaces was examined. Iron coated quartz crystal microbalance (QCM) sensors were used for the experiments, to monitor the deposition of the lipid as well as the acidic corrosion (dissolution) of iron in situ, in real time. The sensors were coated by self-assembled monolayers of di-myristoyl phosphatidic acid using the liposome deposition method. In this process, 50-100 nm vesicles formed by the lipid are delivered in an aqueous solution and spontaneously coat the iron surfaces upon contact. QCM and ellipsometry measurements confirmed that continuous bilayer and monolayer surface coatings can be achieved by this method. QCM measurements also confirmed that the layers were corrosion resistant in $0.01 \mathrm{M}$ acetic acid solution that would dissolve the thin iron layer in minutes in the absence of the protective coating. XPS results suggested a chemisorption-based mechanism of phosphatidic acid attachment to the iron surface. Hence, liposome deposition of phosphatidic acid offers a suitable solution to coat iron surfaces in inaccessible structures in situ.
\end{abstract}

Keywords: Iron, acid corrosion, surfactant coating, self-assembled monolayer, quartz crystal microbalance

'La Trobe Institute for Molecular Science, La Trobe University, Melbourne, Australia

${ }^{2}$ South Gas Company, Ministry of Oil, Iraq

${ }^{*}$ Corresponding author: A. Mechler E-mail: a.mechler@latrobe.edu.au

DOI: 10.2478/ebtj-2019-0015
(C) 2019 Authors. This work was licensed under the Creative Commons AttributionNonCommercial-NoDerivs 4.0 License.

\section{Introduction}

Corrosion is an ever-increasing problem in heavy industries and the resource sector, especially in oil and gas production. The use of inhibitors is one of numerous techniques to prevent or minimize the corrosion of metals especially in acidic media (1-7). Inhibitory action can occur via physical or chemical means, that is, by altering the kinetics of the cathode and/or anode reaction, decreasing the electrical resistance or inhibiting the access of reactants to the metal surfaces (2). Organic inhibitors such as compounds of $\mathrm{P}, \mathrm{N}, \mathrm{O}$, and $\mathrm{S}$ containing aliphatic terminal groups or aromatic rings have been used to protect carbon steel from corrosion in acidic solutions (7-10). Surfactants are a special class of organic inhibitors providing the most effective method to prevent the metal from corrosion (11-16). The advantages of using surfactant inhibitors as anti-corrosion coatings are many: these materials are environmentally friendly, low cost, have a very high inhibition efficiency and are easy to coat on the metal surface (7-17).

Cationic and anionic surfactants are regularly used for preventing corrosion of carbon steel in the presence of carbon dioxide, and imidazoline based cationic surfactants are already extensively used in the petroleum industry corrosion inhibitors because they are not affected by the change of the $\mathrm{pH}$ of the environment $(11,12,14,18)$. However a typical shortcoming of surfactant inhibitors is that they do not fully protect the metal from 
corrosion $(11,12,14,18)$. Hence the application of surfactants as corrosion inhibitors, while an established method, still warrants further studies.

Surfactants coat the metal surfaces due to their amphiphilic character, i.e. via physical interactions. However surface coating can be also chemical, as in the case of self-assembled monolayers. It $s$ well known that monoalkyl phosphates can chemisorb to transition metal oxide surfaces and exhibit some degree of corrosion inhibitory effect when bound to iron surfaces (19). The binding process is chemisorption if hydroxide species are present: iron hydroxide react with the partially protonated phosphate via a condensation reaction $(20,21)$, leading to covalent bonding between the oxygen atoms of the phosphate moiety and the metal in a combination of monodentate, bidentate and tridentate modes. This process is spontaneous at room temperature $(21,22)$. One of the key shortcomings of surface coating with a monoalkyl phosphate self-assembled monolayer is that the packing of the layer is typically imperfect due to oligophosphate formation and the fast formation of chemical bonds prevents compaction of the layer. It is potentially more advantageous to use a complex amphiphile such as phosphatidic acid, where two alkyl chains and a flexible glycerol backbone allow for better packing, especially if delivered to the surface as a pre-formed lamellar assembly, by using the well established liposome deposition method (23-26).

The aim of this work is to investigate the possibility of using di-myristoyl phosphatidic acid (DMPA) as iron anticorrosion coating agent. Phosphatidic acid is environmently friendly, inexpensive, easy to prepare and the double aliphatic hydrocarbon chains may increase the density of the resulting layer to limit water permeability, while the phosphate headgroup may be able to chemisorb to the iron oxide surface.

\section{Materials and Methods}

$20 \mathrm{mM}$ phosphate buffer was prepared with $100 \mathrm{mM}$ sodium chloride ( $\mathrm{NaCl}$, ACS reagent, Merck), dipotassium hydrogen phosphate and potassium dihydrogen phosphate $\left(\mathrm{K}_{2} \mathrm{HPO}_{4}\right.$ and $\mathrm{KH}_{2} \mathrm{PO}_{4}$, respectively; ACS reagent, Fluka, Switzerland). The phosphate buffered saline (PBS) solution was prepared at $\mathrm{pH} 8$ using deionised water $(18.2 \mathrm{M} \Omega \mathrm{cm})$. Acetic acid was diluted with deionised water from glacial acetic acid $99.7 \%$ (ChemSupply, NSW, Australia). Chloroform (ACS Reagent, 99.8\%) was purchased from Sigma Aldrich (Castle Hill, NSW Australia).

\section{Preparation of liposomes}

1,2-dimyristoyl-sn-glycero-3-phosphate (DMPA) was purchased from Avanti Polar Lipids (Alabaster, AL, USA). A stock solution of the DMPA was prepared by dissolving 10 $\mu \mathrm{mol}(6.15 \mathrm{mg})$ of the DMPA in $750 \mu \mathrm{l}$ chloroform and 250 $\mu \mathrm{l}$ methanol. Then $0.5 \mu \mathrm{mol}$ lipid was measured out into a glass test tube, dried under a gentle stream of nitrogen gas and placed in a desiccator overnight. Then it was dissolved in $1 \mathrm{ml}$ PBS and gently sonicated to form a suspension of spherical bilayer vesicles for surface modification in the QCM.

\section{Quartz crystal microbalance corrosion experiments}

Mass loss experiments were conducted using a Q-Sense E1 instrument (Q-Sense AB, Gothenburg, Sweden). Iron coated sensor chip QSX 319 with $100 \mathrm{~nm}$ Fe layer (nominal), packed in inert gas, was purchased from the same company. Substrate is an AT cut quartz crystal with a fundamental resonance frequency of $5 \mathrm{MHz}$. The frequency shift $(\Delta f)$ and energy dissipation ( $\Delta$ $D$ ) were recorded at the $3^{\text {rd }}, 5^{\text {th }}, 7^{\text {th }}, 9^{\text {th }}, 11^{\text {th }}$ and $13^{\text {th }}$ eigenmodes; reported sensograms are for the 5 th eigenmode. Corrosion as mass loss (dissolution of iron) was measured with nanogram accuracy using acetic acid (HAc) as the corrosive agent in aqueous solution. $0.1 \mathrm{M}$ and $0.01 \mathrm{M} \mathrm{HAc}$ solutions were used at $25^{\circ} \mathrm{C}$ in situ in the QCM chamber. Reference sensograms were recorded in deionised water $(18.2 \mathrm{M} \Omega \mathrm{cm})$ for $24 \mathrm{hrs}$.

Lipid deposition was performed in the QCM chamber in situ by introducing $0.5 \mu \mathrm{mol}$ of DMPA suspended in $1 \mathrm{ml}$ PBS. PBS was used to improve the stability of the liposomes used for deposition (27-29). First the chip was cleaned with deionized water and dried with nitrogen gas before assembling into the QCM chamber. For a typical experiment, water was flushed in the chamber for at least $5 \mathrm{~min}$. A water baseline was recorded before introducing the PBS. After a PBS baseline DMPA liposome suspension was introduced and the frequency and the dissipation change was recorded in real time. Finally, a PBS wash was used to achieve the final baseline that was used to determine the amount of lipid attached to the surface. Experiments were repeated at least 3 times (i.e. $n=3$ ).

Adsorbed/removed mass and the oxidation rate were calculated using the Sauerbrey equation (30)

$$
\Delta m=-C \frac{\Delta f}{n}
$$

where $\Delta m$ is the adsorbed mass in $\mathrm{ng} / \mathrm{cm}^{2}, \mathrm{C}$ is a constant (17.7 $\mathrm{ng} \mathrm{cm}^{-2} \mathrm{~Hz}^{-1}$ at $\mathrm{f}=5 \mathrm{MHz}$, the eigenfrequency of the unloaded crystal) and $\Delta \mathrm{f}$ is the change in frequency at $\mathrm{n}$ overtone of the crystal oscillation.

\section{X-Ray Photoelectron Spectroscopy}

The chemical composition of the oxidized iron surface was characterized by X-Ray Photoelectron Spectroscopy (XPS) using a Kartos Axis Nova spectrometer (Kartos Analytical, Manchester, United Kingdom). A monochromatic $\mathrm{Al} \mathrm{K}_{\alpha}$ source $(10 \mathrm{~mA}, 15 \mathrm{kV})$ was used at $7 \times 10^{-9}$ Torr pressure. The data were analysed using CasaXPS (version 2.3.15) and Origin Pro 2015 software.

The analysis of the iron $2 \mathrm{p}_{3 / 2}$ peak is particularly challenging due to the series of satellite peaks present in each oxidation state, necessitating the use of synthetic model functions formed from a series of peaks (31-33). Given that on an oxidized iron surface at least three oxidation states are likely present with potential shifts due to changing chemical environment, quantitative analysis is not possible; however a qualitative assessment of the iron species can be attempted (34-35) For this we followed the data published by Biesinger et $a^{33}$ that includes peak parameters for $\mathrm{FeOOH}$ (Table 1) to fit the experimental data using the 
Table 1. The XPS parameters: peak position (eV), FWHM, and the intensities for fitting the Fe $2 p_{3 / 2}$; data from (33). Ave is the average value of the respective $\mathrm{a}$ and $\gamma$ components of $\mathrm{Fe}_{2} \mathrm{O}_{3}$ and $\mathrm{FeOOH}$.

\begin{tabular}{|c|c|c|c|}
\hline Compound & Peak (eV) & FWHM & $\%$ \\
\hline $\mathrm{Fe}$ & 706.6 & 0.88 & 100.0 \\
\hline $\mathrm{FeO}(1)$ & 708.4 & 1.4 & 24.2 \\
\hline $\mathrm{FeO}(2)$ & 709.7 & 1.6 & 30.1 \\
\hline $\mathrm{FeO}(3)$ & 710.9 & 1.6 & 14.5 \\
\hline $\mathrm{FeO}(4)$ & 712.1 & 2.9 & 25.6 \\
\hline $\mathrm{FeO}(5)$ & 715.4 & 2.5 & 5.6 \\
\hline Ave. $\mathrm{Fe}_{2} \mathrm{O}_{3}(1)$ & 709.8 & 1.1 & 26.8 \\
\hline Ave. $\mathrm{Fe}_{2} \mathrm{O}_{3}(2)$ & 710.8 & 1.3 & 24.7 \\
\hline Ave. $\mathrm{Fe}_{2} \mathrm{O}_{3}(3)$ & 711.6 & 1.3 & 18.9 \\
\hline Ave. $\mathrm{Fe}_{2} \mathrm{O}_{3}(4)$ & 712.7 & 1.4 & 10.1 \\
\hline Ave. $\mathrm{Fe}_{2} \mathrm{O}_{3}(5)$ & 713.7 & 2.0 & 10.0 \\
\hline Ave. $\mathrm{FeOOH(1)}$ & 710.3 & 1.4 & 27.0 \\
\hline Ave. $\mathrm{FeOOH(2)}$ & 711.3 & 1.3 & 26.5 \\
\hline Ave. $\mathrm{FeOOH}(3)$ & 712.2 & 1.4 & 20.6 \\
\hline Ave. $\mathrm{FeOOH}(4)$ & 713.3 & 1.4 & 11.3 \\
\hline Ave. $\mathrm{FeOOH}(5)$ & 714.1 & 1.9 & 6.3 \\
\hline
\end{tabular}

CasaXPS software. In this approach the peak positions, FWHM and relative intensities of the peak series for each species are fixed; only the intensities of the entire series are altered to fit the experimental data. As the table shows, there is a distinct shift between the $\mathrm{Fe}_{2} \mathrm{O}_{3}$ and $\mathrm{FeOOH}$ peak series that allows for qualitative assessment of the presence of the three iron species.

\section{Ellipsometry measurements}

Layer thicknesses were measured using a Picometer Ellipsometer (Beaglehole Instruments, New Zealand). All measurements were conducted on dried samples. Variable angle scan method was used at $\lambda=633.0 \mathrm{~nm}$ wavelength for measuring the thickness of iron oxide. There are no dielectic parameters for DMPA in the database, therefore a material file was created based on Cauchy model (i.e. dielectric material). The Cauchy parameters were calculated from spectroscopic measurements on lipids at a wavelength range of 400-800 $\mathrm{nm}$ at 67 degrees incidence angle. The thicknesses of the DMPA layers were measured based on angle scans at $550 \mathrm{~nm}$ wavelength. Fitting for layer thickness was conducted in Thin Film Companion (TFC) software (Semiconsoft).

\section{Results}

\section{Benchmarking the acidic corrosion of the iron surface}

To establish a reference, corrosion of iron in acetic acid was monitored using QCM. Experiments were performed with
$0.1 \mathrm{M}$ and $0.01 \mathrm{M}$ acetic acid at $25^{\circ} \mathrm{C}$, and the rates of iron dissolution were established. Fig. 1. shows and example of the experimental results, the effect of $10 \mathrm{mM}$ acetic acid on the iron surface at $25^{\circ} \mathrm{C}$. After an initial period ( $\left.\sim 8 \mathrm{~min}\right)$ linear increase of the frequency from zero to $2.3 \mathrm{kHz}$ is observed, indicating substantial mass loss, when the acid was flushed with deionized water to stop further dissolution. The initial resistance of the layer to dissolution is consistent with the presence of a thin passivating oxide layer that was breached at about $8 \mathrm{~min}$. This pattern was observed in all corrosion experiments, with some variation of the time needed for activation. The rate of corrosion is a function of several factors, including temperature, acid concentration, as well as surface roughness of the iron coating and the thickness and composition of the surface oxide layer; accordingly the measured rate was varied. At $25^{\circ} \mathrm{C}$ for $0.01 \mathrm{M}$ HAc solution it was typically $\sim 6 \mathrm{~Hz} / \mathrm{min}$, or $\sim 0.1 \mathrm{mg} / \mathrm{min}$, and for $0.1 \mathrm{M} \mathrm{HAc}$ concentration the rate increased to $\sim 400 \mathrm{~Hz} / \mathrm{min}$ or $\sim 7.1 \mathrm{mg} / \mathrm{min}$. This is a suitable reference for the qualitative study of corrosion inhibition.

\section{Lipid coating of the iron surface}

DMPA was deposited on the iron coated sensor chip surface using the liposome deposition method. Experiments were conducted at $25{ }^{\circ} \mathrm{C}$. A typical sensogram is shown in Fig. 2 . First deionised water was introduced into the chamber until the sensor signals stabilized. Next PBS was introduced for $10 \mathrm{~min}$ 


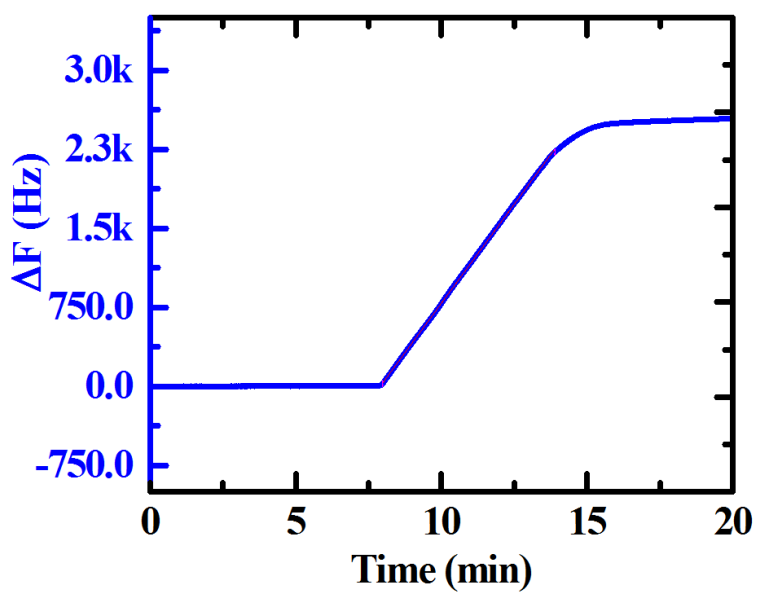

Figure 1. Representative QCM frequency sensogram of the corrosion experiment showing dissolution of iron at $25^{\circ} \mathrm{C}$ using $0.01 \mathrm{M}$ acetic acid solution. Ultrapure water was flushed through the chamber at $13 \mathrm{~min}$ to stop corrosion.

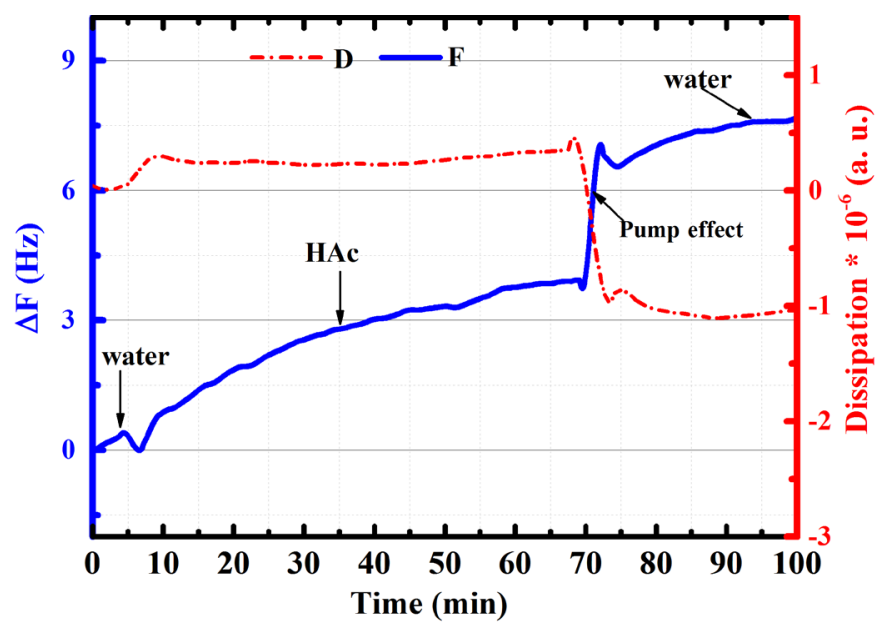

Figure 3. Representative QCM sensogram showing the removal of the top membrane leaflet by acetic acid on the DMPA coated iron chip.

to obtain a baseline. This was followed by the DMPA liposome suspension that lead to a sudden decrease of frequency, indicating mass uptake, as the DMPA liposomes attached to the surface. PBS rinse was applied after the DMPA deposition to remove excess liposomes, followed by osmotic stress treatment with pure water to burst open any remaining liposomes. PBS was reintroduced to measure the final mass of the deposit layer. The frequency change for surface bound lipid was $\boldsymbol{\Delta} f$ $\sim-20 \mathrm{~Hz}$ in PBS; this value is consistent with a single bilayer surface coating of a tightly bound layer (25-26). 0.01 M acetic acid was introduced at $1.7 \mathrm{hrs}$; the slight change in frequency reflects the different viscosity of the acetic acid solution (i.e. absence of hydrated ions of the $\mathrm{NaCl}$ ). It is clear that no mass loss was recorded over one hour, unlike in the benchmarking experiments where acetic acid started dissolving the iron chip within minutes. Hence the DMPA bilayer coverage on the surface inhibited corrosion.

In some of the experiments a slow baseline drift was observed

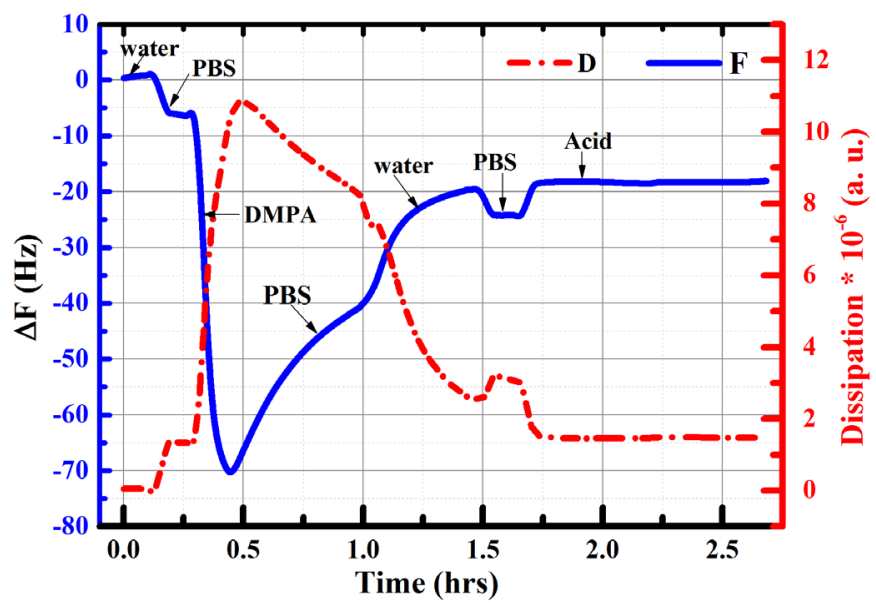

Figure 2. A typical QCM sensogram of a DMPA deposition and corrosion inhibitory test as changes of frequency $(\Delta f$; solid blue line) and energy dissipation ( $\triangle D$; dash red line multiplied by $10^{-6}$ ) against the time.

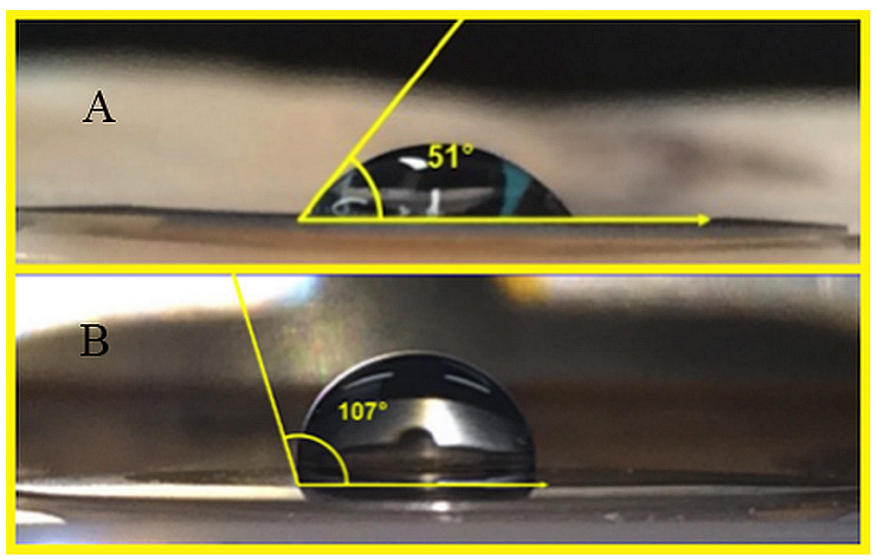

Figure 4. Contact angles of a water droplet on (A) uncoated iron oxide and (B) lipid coated iron oxide surface.

upon addition of the acid solution, which slowed down over time, asymptotically approaching a stable value (Fig. 3). That and the magnitude of the change $(\sim 4 \mathrm{~Hz})$ rules out corrosion as the cause; it is more likely that the top leaflet of the lipid bilayer is removed, parallel with further compaction of the bottom (surface bound) leaflet. If this is the case, the exposed surface has to be hydrophobic, opposed to the bilayer with the exposed phosphate groups that is hydrophilic. Hydrophobicity of the coating offers further advantage in restricting water access and reducing viscous drag in applications.

\section{Contact Angle Measurements}

To confirm that the resulting surface is hydrophobic as expected if a single leaflet membrane is present, contact angle measurements were performed before and after the coating. A water drop was stabilized on the chip surface at room temperature and the contact angles were measured as in Figure 4. Before the coating, the water drop preferentially wetted the 


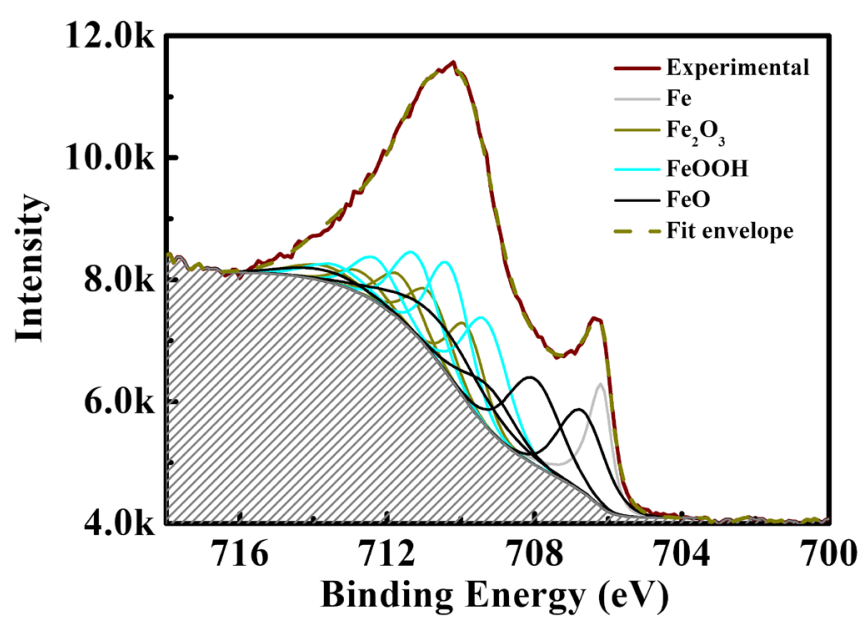

Figure 5. Fitted XPS spectrum of Fe $2 \mathrm{p}_{3 / 2}$ for the mixed iron oxide surface layer of the iron coated QCM chip using CasaXPS software (plotted in OriginPro). For fitting the method by by Biesinger et al (33) was used where the peak positions, FWHM and relative peak intensities were maintained constant for each series of peaks representing the specific oxidation states of iron; only the peak heights of the entire series were varied in the fitting.

iron oxide and the measured contact angle was $\sim 51^{\circ}$ (Fig. $4 \mathrm{~A}$ ). After coating and exposure to acetic acid, the contact angle was about $107^{\circ}$ as the water did not spread on the surface (Fig. 4 B).

In the hydrophobic case, the surface energy can be calculated. The work of adhesion is related to the contact angle by the Young-Dupré equation (1) (36-37).

$$
\mathrm{W}_{\mathrm{sl}}=\gamma_{1}(\cos \theta+1)
$$

where $\gamma_{1}$ is the surface tension of water, $\theta$ is the contact angle. The methyl and methylene moieties of the lipid phase are apolar with low polarizability, therefore even at the water interface the contribution of polar interactions to the total work of adhesion can be neglected. In the absence of polar interactions the work of adhesion at an interface can be also approximated from the surface free energies of the materials that form the interface:

$$
\mathrm{W}_{\mathrm{sl}} \approx \sqrt{ } \mathrm{W}_{\mathrm{ss}}^{\mathrm{d}} \mathrm{W}_{\mathrm{ll}}^{\mathrm{d}} \approx 2 \sqrt{ } \gamma_{\mathrm{s}}^{\mathrm{d}} \gamma_{1}^{\mathrm{d}} \ldots \ldots \ldots
$$

where $\gamma^{\mathrm{d}}{ }_{\mathrm{s}}$ and $\gamma_{1}^{\mathrm{d}}$ are thedispersion force contributions to

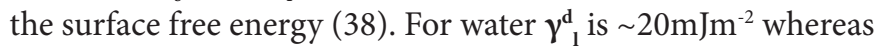
for apolar materials, such as hydrocarbons, $\boldsymbol{\gamma}_{\mathrm{s}}^{\mathrm{d}}$ makes up $100 \%$ of the surface free energy. Hence, for the water-lipid interface this approximation provides a reliable estimate. By substituting equation (2) in the Young-Dupré eq (1) it is possible to calculate the surface tension of the solid-vapor ( $\sim$ solid-vacuum) interface, that is, the surface energy of the solid:

$$
\gamma_{l}=\frac{\gamma_{l}^{2}}{4 \gamma_{1}^{\mathrm{d}}}(\cos \theta+1)^{2}
$$

Hence the surface free energy of the lipid monolayer-coated surface is $\sim 32,4 \mathrm{mJm}^{-2}\left(\theta=107^{\circ}\right)$ which is comparable to the surface energies of bulk hydrocarbons (e.g. for octadecane $\gamma=28 \mathrm{mJm}^{-2}$ ). The same approximation cannot be applied to the hydrophilic case. The low surface free energy confirms the removal of the top leaflet of the lipid bilayer, leaving the alkyl chains exposed.

\section{Ellipsometry results}

As an additional confirmation of the thickness of the DMPA coating, ellispometry was used. First the thickness of the iron oxide layer was determined after oxidation with acetic acid. The measurements were done on the same QCM chips used for the corrosion experiments. Varied angle method was used to measure the thickness at $633 \mathrm{~nm}$ wavelength. The thickness of the iron oxide was measured to be $5.5 \mathrm{~nm}$ and this value was then used to parametrize the layer stack for the measurements on DMPA.

The thicknesses of the lipid layers were measured before and after exposure to acetic acid. The measured ellipsometric thickness of the lipid deposit before acid treatment was $4.2 \mathrm{~nm}$, which is consistent with the thickness of a DMPA bilayer. The thickness after acid treatment was reduced to $2.0 \mathrm{~nm}$ which is close to the theoretical and practical value of the self-assembled monolayer of C14-PA (39). This result is further proof that upon exposure to acetic acid a monolayer of DMPA remained on the iron oxide surface.

\section{XPS measurements}

The formation of phosphate-based self-assembled monolayers on transition metal oxide surfaces is based on a condensation reaction between the phosphate moiety and hydroxyl groups on the surface $(32,42)$. It can be assumed that a hydrated metal oxide surface contains hydroxyl moieties; to gain more certainty the surface of an iron coated sensor was characterized by XPS. A representative spectrum of the iron $2 \mathrm{p}_{3 / 2}$ peak is shown in Fig. 5 (black line).

Synthetic model functions have been constructed from the characteristic peak series of $\mathrm{FeO}, \mathrm{Fe}_{2} \mathrm{O}_{3}$ and $\mathrm{FeOOH}$ in CasaXPS that also employs a range of built-in factors to perform peak 


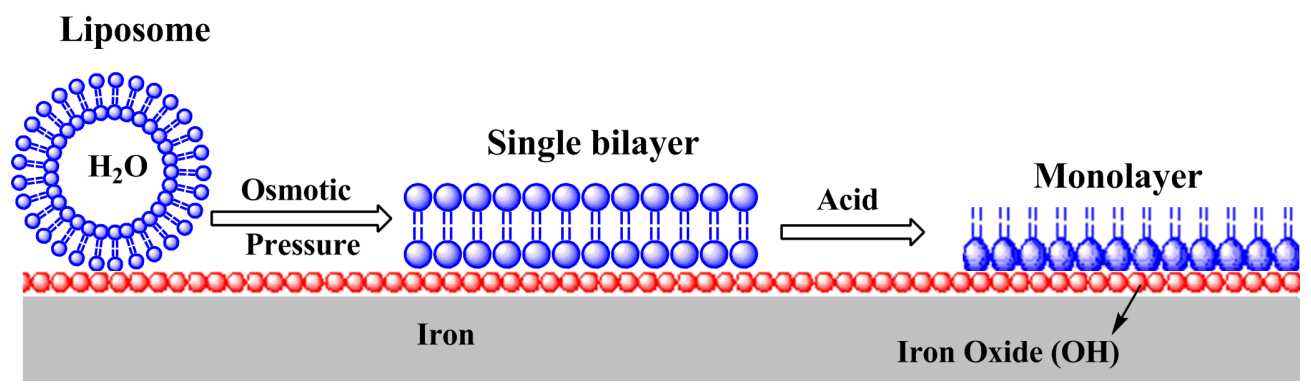

Figure 6. The mechanism of surface coating by liposome deposition. Bilayer coating forms spontaneously upon contact. The top leaflet of the bilayer may be removed upon acid treatment, however the bottom leaflet remains stable on the surface.

fitting optimized for XPS data analysis. As stated above, in the fit only the intensities of the peak series (i.e. 4 parameters) were fitted: within each series the peak positions, FWHM and relative peak intensities were maintained constant. None of the composite functions could fit the peak accurately when combined only with the $\mathrm{Fe}^{0}$ peak. Therefore the analysis of the spectra confirms qualitatively the presence of iron hydroxide besides the $\mathrm{Fe}^{2+}$ and $\mathrm{Fe}^{3+}$ oxide species.

\section{Discussion}

According to the QCM measurements the formation of the lipid coating is very fast. In contrast, the self-assembly of monolayers such as thiols on gold is generally a very slow process (41$44)$, where longer time produces better monolayer packing. However, DMPA is delivered to the surface in bilayer vesicles where the packing is already at energy minimum following the Israelachvili-Mitchell-Ninham model of surfactant selfassembly $(45,46)$. That raises the possibility that a tightly packed supported layer may form instantaneously upon the rupture of the vesicles.

Based on the data the mechanism of surface coating follows the steps shown in Fig. 6. DMPA vesicles (liposomes) delivered in a PBS environment attach to the surface upon contact. When treated with pure water, osmotic pressure bursts the liposomes and the bilayers form spontaneously. The bilayers are stable
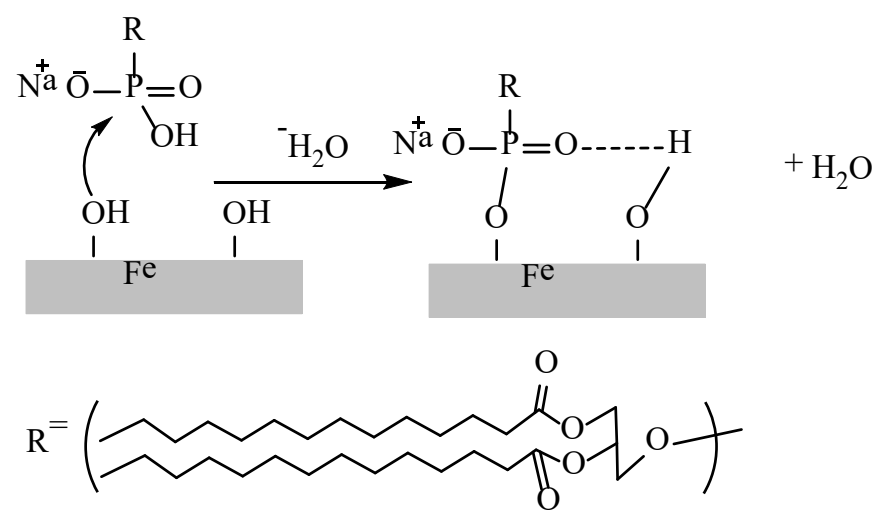

Figure 7. The condensation reaction mechanism of the lipid on the iron oxide surface. The hydroxyl group of the iron attaches to the phosphoryl group and the consequence of this step is chemical binding of the lipid to the metal oxide. when removed from the aqueous environment, likely due salt formation between the charged moiety of the top leaflet lipids and small counterions from the assay buffer. However, after exposed to mild acidic conditions, in some cases the top leaflet of the bilayer is removed as shown by QCM and ellipsometry.

The removal of the top leaflet as a physical process is energetically unfavourable due to the very high energy cost of the exposure of the membrane core in the aqueous environment. It is also notable that it only takes place in HAc environment. Therefore it is likely a chemical process, that is, acidic hydrolisis of the ester groups of the lipid molecules that leads to disintegration and dissolution of the top leaflet. The surface attached bottom leaflet remains stable due to the impermeability of the apolar membrane core to any ionic species, including the acid. The high stability of the bottom leaflet in the aqueous medium suggests that the attachment of the phosphate groups to the iron surface is chemisorption.

Iron hydroxide can react with the partially protonated phosphatidic acid via a condensation reaction as described in the introduction (20-21). In this process the phosphoryl oxygen of the PA coordinate to the Lewis acidic metal oxide followed by a heterocondensation with the hydroxylated surface, or the hydroxyl group of the surface binds to the phosphoryl group and eliminate water (Fig. 7). It is likely that the templating effect of the energy minimized bilayer of the liposomes leads to the fusion of a largely continuous layer, as opposed to the generally much less ordered phosphate SAMs. This is consistent with the absence of any iron dissolution in HAc after the PA surface modification.

\section{Conclusions}

A method of coating a phosphatidic acid corrosion inhibitory layer on iron metal by liposome deposition has been developed. The base rate of corrosion was established using acetic acid solution at different temperatures and concentrations where the weight loss was monitored by using QCM technique. Phosphatidic acid coating (DMPA) was formed on the iron oxide with the liposome deposition method and the presence of the coating was confirmed by QCM. The DMPA layer has successfully inhibited corrosion. After treatment with acetic acid, it was noted that a lipid monolayer has remained on the iron surface. This was confirmed by contact angle measurements that have shown that the coated surface is hydrophobic, and 
ellipsometry results that showed a decrease of the thickness of a lipid layer from $4.2 \mathrm{~nm}$ (bilayer) to $2.0 \mathrm{~nm}$ (monolayer). XPS was used to study the iron oxide chemistry and shows the presence of hydroxide on the hydrated surface, hence the mechanism of adsorption of lipid on the metal surface was identified as a covalent binding between the PA moiety and the metal. The DMPA layer had a good corrosion inhibitory effect due to the strong van der Waals force between the hydrocarbon chains, which prevent the water from reaching the metal surface.

\section{Acknowledgments}

A. S. A.-B. isgrateful to HCED (The Higher Committee for Education Development in Iraq) for the financial support of a scholarship. Also, we would like to acknowledge Dr. Rob Jones from the Centre of Material and Surface Science (CMSS) for performing the XPS experiment and for his explanation of the XPS data analysis principles and the use of the CasaXPS software.

\section{Conflict of Interest}

The authors declare that they have no conflicts of interest.

\section{Ethical Compliance}

This article does not contain any studies involving human participants or animals performed by any of the authors.

\section{References}

1. Jevremović l, Singer $M$, Nešić S and Mišković-Stanković V, Corrosion Science, 2013, 77, 265-272.

2. Raja PB and Sethuraman MG, Materials Letters, 2008, 62, 113-116.

3. Soliman SA, Metwally MS, Selim SR, Bedair MA and Abbas MA, Journal of Industrial and Engineering Chemistry, 2014, 20, 43114320.

4. Shpan'ko SP, Grigor'ev VP, Anisimova VA, Plekhanova EV and Tolpygin IE, Protection of Metals and Physical Chemistry of Surfaces, 2013, 49, 859-864.

5. El Bribri A, Tabyaoui M, Tabyaoui B, El Attari H and Bentiss F, Materials Chemistry and Physics, 2013, 141, 240-247.

6. Moussa M, El-Far A and El-Shafei A, Materials chemistry and physics, 2007, 105, 105-113.

7. Fuchs-Godec R and Dolecek V, Colloids and Surfaces a-Physicochemical and Engineering Aspects, 2004, 244, 73-76.

8. Obot I and Obi-Egbedi N, Current Applied Physics, 2011, 11, 382392.

9. Bouklah M, Benchat N, Aouniti A, Hammouti N, Benkaddour M, Lagrenée $M$, Vezin $\mathrm{H}$ and Bentiss $F$, Progress in organic coatings, 2004, 51, 118-124.

10. Babu BR and Thangavel K, Anti-Corrosion Methods and Materials, 2005, 52, 219-225.

11. Butler LN, Fellows CM and Gilbert RG, Progress in Organic Coatings, 2005, 53, 112-118.

12. Deyab MA, International Journal of Hydrogen Energy, 2013, 38, 13511-13519.

13. Qiu LG, Xie AJ and Shen YH, Corrosion science, 2005, 47, 273-278.

14. Starovoitova YV, Andreev NN, Gedvillo IA and Zhmakina AS, Protection of Metals and Physical Chemistry of Surfaces, 2009, 45, 792-795.

15. Tawfik SM and Zaky MF, Research on Chemical Intermediates, 2015, 41, 8747-8772.
16. Tamilarasan TR, Rajendran R, Rajagopal R and Sudagar J, Surface \& Coatings Technology, 2015, 276, 320-326.

17. Murira CM, Punckt C, Schniepp HC, Khusid and Aksay IA, Langmuir, 2008, 24, 14269-14275.

18. Amalhay M and Ignatiadis I, Materials science forum, 1998.

19. Gao X, Liu ST, Lu HF, Gao F and Ma HY, Industrial \& Engineering Chemistry Research, 2015, 54, 1941-1952.

20. Dettin M, Bagno A, Gambaretto R, lucci G, Conconi MT, Tuccitto N, Menti AM, Grandi C, Di Bello C, Licciardello A and Polzonetti G, Journal of Biomedical Materials Research Part A, 2009, 90A, 3545.

21. Pujari SP, Scheres L, Marcelis ATM and Zuilhof $\mathrm{H}$, Angewandte Chemie-International Edition, 2014, 53, 6322-6356.

22. Hotchkiss PJ, Jones SC, Paniagua SA, Sharma A, Kippelen B, Armstrong NR and Marder SR, Accounts of Chemical Research, 2012, 45, 337-346.

23. Richter R, Mukhopadhyay A and Brisson A, Biophysical Journal, 2003, 85, 3035-3047.

24. Richter R, Berat $R$ and Brisson AR, Langmuir, 2006, 22, 3497-3505.

25. Hasan IY and Mechler A, Soft Matter, 2015, 11, 5571-5579.

26. Mechler A, Praporski S, Piantavigna S, Heaton SM, Hall KN, Aguilar MI and Martin LL, Biomaterials, 2009, 30, 682-689.

27. Cho NJ, Frank CW, Kasemo B and Hook F, Nature Protocols, 2010 , 5, 1096-1106.

28. J. A. Jackman, S. R. Tabaei, Z. L. Zhao, S. Yorulmaz and N. J. Cho, Acs Applied Materials \& Interfaces, 2015, 7, 959-968.

29. Tabaei SR, Choi JH, Zan GH, Zhdanov VP and Cho NJ, Langmuir, 2014, 30, 10363-10373.

30. Stålgren J, Eriksson J and Boschkova $\mathrm{K}$, Journal of colloid and interface science, 2002, 253, 190-195.

31. Yamashita T and Hayes P, Applied Surface Science, 2008, 254, 2441-2449.

32. A. Grosvenor, B. Kobe, M. Biesinger and N. McIntyre, Surface and Interface Analysis, 2004, 36, 1564-1574.

33. Biesinger MC, Payne BP, Grosvenor AP, Lau LW, Gerson AR and Smart RSC, Applied Surface Science, 2011, 257, 2717-2730.

34. Mcintyre NS and Zetaruk DG, Analytical Chemistry, 1977, 49, 1521-1529.

35. Pratt AR, Muir IJ and Nesbitt HW, Geochimica Et Cosmochimica Acta, 1994, 58, 827-841.

36. Rosen MJ and Kunjappu JT, Surfactants and interfacial phenomena, John Wiley \& Sons, 2012.

37. Israelachvili JN, Intermolecular and surface forces: revised third edition, Academic press, 2011.

38. Kwok DY and Neumann AW, Colloids and Surfaces A, 2000, 161, 31-48.

39. Khassanov A, Steinrück HG, Schmaltz T, Magerl A and Halik M, Accounts of Chemical Research, 2015, 48, 1901-1908.

40. McCafferty E and Wightman J, Surface and Interface Analysis, 1998, 26, 549-564.

41. Shaporenko A, Rossler K, Lang $\mathrm{H}$ and Zharnikov M, Journal of Physical Chemistry B, 2006, 110, 24621-24628.

42. Fajin JLC, Teixeira F, Gomes JRB and Cordeiro M, Theoretical Chemistry Accounts, 2015, 134.

43. Vericat C, Vela ME, Benitez G, Carro P and Salvarezza RC, Chemical Society Reviews, 2010, 39, 1805-1834.

44. Wang XY, Liu YS, Chen ZL, Li Y, Sun K and Jiang XY, Journal of Materials Science, 2014, 49, 4394-4398.

45. Israelachvili JN, Mitchell DJ and Ninham BW, Biochimica Et Biophysica Acta, 1977, 470, 185-201.

46. Israelachvili JN, Mitchell DJ and Ninham BW, Journal of the Chemical Society-Faraday Transactions li, 1976, 72, 1525-1568. 